\section{Sur la sommabilité des séries de Fourier}

par

Z. ZALCWASSER (W'arszawa).

1. Soit

$$
\frac{a_{0}}{2}+\sum_{k=1}^{\infty}\left(a_{k} \cos k x+b_{k} \sin k x\right)
$$

la série de Fourier d'une fonction sommable $f(x)$ et

$$
S_{0}(x), \quad S_{1}(x), \quad S_{2}(x), \ldots
$$

la suite des sommes partielles de la série (1). D'après un théorème classique de $\mathrm{M}$. H. Lebesgue, la suite (2) est presque partout sommable $(\mathrm{C}, 1)$ vers la fonction $f(x)$. Or, comme la suite (2) n'est pas en général convergente, on peut se poser le problème suivant: Formons une suite

$$
S_{p_{1}}(x), \quad S_{p_{2}}(x), \ldots . \quad\left(p_{1}<p_{2}<\ldots\right),
$$

extraite de la suite (2). La nouvelle suite (3) est-elle encore sommable $(C, 1)$ presque partout? Nous avons obtenu sur ce sujet deux résultats particuliers.

2. Théorème I. $f(x)$ étant une fonction sommable, on a presque partout

$$
\lim _{n \rightarrow \infty} \frac{1}{n}\left[S_{1^{2}}(x)+S_{2^{2}}(x)+\ldots+S_{n^{2}}(x)\right]=f(x) .
$$

Démonstration. On a

(4) $\frac{1}{n} \sum_{r=1}^{n} S_{v 2}(x)-f(x)=\frac{1}{n \pi} \int_{0}^{\pi} \varphi_{x}(t) \frac{H_{n}(t)}{2 \sin \frac{1}{2} t} d t=I_{n}$,

où
(5) $\varphi_{x}(t)=f(x+t)+f(x-t)-2 f(x) ; H_{n}(t)=\sum_{n=1}^{n} \sin \left(\nu^{2}+1 / 2\right) t$

$$
\left|H_{n}(t)\right| \leqslant\left|\sum_{i=1}^{n} e^{i\left(\gamma^{2}+1 / 2\right) t}\right|=\left|\sum_{v=1}^{n} e^{i v^{2} t}\right|
$$

Pour obtenir une borne supérieure des sommes $\left|\sum e^{i v^{2} t}\right|$, on peut appliquer le lemme de $M$. van der Corput $^{1}$ ):

Si $g(u)(a \leqslant u \leqslant b)$ est une fonction réelle deux fois dérivable et si $g^{\prime \prime}(u) \geqslant \varrho>0$ pour $a \leqslant u \leqslant b$ ( $\varrho$ - une constante), alors

$A$ étant une constante absolue.

$$
\left|\sum_{a<v \leq b} e^{2 \pi i g(v)}\right| \leqslant\left[g^{\prime}(b)-g^{\prime}(a)+2\right]\left(\frac{4}{\sqrt{\varrho}}+A\right),
$$

Ici nous avons

$$
g(u)=\frac{u^{2} t}{2 \pi}, \quad g^{\prime}(u)=\frac{u t}{\pi}, \quad \rho=\frac{t}{\pi} \quad(t>0), a=0, b=n
$$

donc

$$
\left|\sum_{n=1}^{n} e^{i \gamma^{2} t}\right| \leqslant\left(\frac{n t}{\pi}+2\right)\left(\frac{4 \sqrt{\pi}}{\sqrt{t}}+A\right)
$$

Revenons à l'expression (4); décomposons l'intégrale $I_{n}$ de la manière suivante:

$$
I_{n}=\frac{1}{n \pi}\left[\int_{0}^{1 / n^{2}}+\int_{1 / n^{2}}^{1 / n}+\int_{1 / n}^{\delta}+\int_{d}^{\pi}\right]=U_{1}+U_{2}+U_{3}+U_{4},
$$

désignons

$$
\int_{0}^{h}\left|\varphi_{x}(t)\right| d t=\Phi_{x}(h)=\Phi(h)
$$

et supposons que

$$
\left.\Phi^{\prime}(0)=0, \quad \text { c. à. d. } \Phi(h)=o(h)^{2}\right),
$$

1) J. G. van der Corput, Zahlentheoretische Abschätzungen, Math. Ann. 84 (1921) p. $53-79$.

2) Il est bien connu que cette hypothèse est remplie par presque tout $x$.
esgue). 
alors nous aurons

$$
\begin{gathered}
(10)_{1} \quad\left|U_{1}\right| \leqslant \frac{1}{n \pi} \int_{0}^{1 / n^{2}} \frac{\left|\varphi_{x}(t)\right|}{2 \sin \frac{1}{2} t} \sum^{n} \sum^{\prime}\left(\nu^{2}+1 / 2\right) t d t \\
\leqslant \frac{1}{n \pi} \int_{0}^{1 / n^{2}}\left|\varphi_{x}(t)\right| 2 n^{3} d t=\frac{2}{\pi} n^{2} \Phi\left(1 / n^{2}\right)=o(1) .
\end{gathered}
$$

Dans l'intégrale $U_{2}$ on a $n t \leqslant 1$ et l'inégalité (7) se simplifie:

$$
\left|H_{n}(t)\right| \leqslant\left|\sum_{n=1}^{n} e^{i, y^{\mathrm{a}} t}\right| \leqslant \frac{A_{1}}{\sqrt{t}} \quad\left(0<t \leqslant \frac{1}{n}\right),
$$

$A_{1}$ étant une constante absolue; il s'ensuit que

$$
\begin{aligned}
\left|U_{2 n}\right| \leqslant \frac{1}{n \pi} \int_{1 / n^{2}}^{1 / n}\left|\varphi_{x}(t)\right| \frac{2 A_{1}}{t^{3 / 2}} d t & =\frac{2 A_{1}}{n \pi}\left\{\left[\Phi(t) t^{-3 / 2}\right]_{1 / n^{2}}^{1 / n}+\frac{3}{2} \int_{1 / n^{2}}^{1 / n} \Phi(t) t^{-5 / 2} d t\right\} \\
& =U_{2}^{\prime}+U_{2}^{\prime \prime} .
\end{aligned}
$$

De l'hypothèse (9) il résulte immédiatement que $U_{2}^{\prime}=o(1)$ et

donc

$$
U_{2}^{\prime \prime}=\frac{o(1)}{n} \int_{1 / n^{2}}^{1 / n} t^{-3 / 2} d t=o(1)
$$

$(10)_{2}$

$$
U_{2}=o(1) \text {. }
$$

Dans l'intégrale $U_{3}$ on a $n t \geqslant 1$ et de l'inégalité (7) on déduit (7) $)_{2}\left|\sum_{n=1}^{n} e^{i y^{2} t}\right| \leqslant A_{2} n \sqrt{t} \quad\left(A_{2}\right.$ - une constante absolue; $\left.t \geqslant \frac{1}{n}\right)$,

$$
\left|U_{3}\right| \leqslant \frac{1}{n \pi} \int_{1 / n}^{\delta}\left|\varphi_{x}(t)\right| \frac{2 A_{2} n}{\sqrt{t}} d t=\frac{2 A_{2}}{\pi}\left\{\left[\Phi(t) t^{-1 / 2}\right]_{1 / n}^{\delta}+\frac{1}{2} \int_{1 / n}^{\delta} \Phi(t) t^{-3 / 2} d t\right\}
$$$$
=U_{3}^{\prime}+U_{3}^{\prime \prime} \text {. }
$$

On vérifie aisément que

$$
\left|U_{3}^{\prime}\right| \leqslant M \text { y } \bar{\delta}, \quad\left|U_{3}^{\prime \prime}\right| \leqslant M \sqrt{\delta}
$$

$M$ ne dépendant ni de $n$ ni de $\delta$ on peut choisir $\delta$ de manière que l'on ait

$(10)_{3}$

$$
\left|U_{3}\right|<\varepsilon \text { pour tous les } n>\frac{1}{\delta} \text {. }
$$

En tenant fixe le nombre $\delta$ démontrons enfin que $\lim _{n \rightarrow \infty} U_{4}=0$. A cet effet il suffit de remarquer que

$$
U_{4}=\frac{v_{1}+v_{2}+. .+v_{n}}{n} \text {, où } v_{n}=\frac{1}{\pi} \int_{0}^{\pi} \frac{\varphi_{x}(t)}{2 \sin \frac{1}{2} t} \sin \left(\nu^{2}+1 / 2\right) t d t \text {; }
$$

la fonction $\frac{\varphi_{x}(t)}{2 \sin \frac{1}{2} t}$ étant sommable dans l'intervalle $(\delta, \pi)$, on a $\left.v_{n} \rightarrow 0^{9}\right)$ et $\grave{a}$ fortiori

$$
(10)_{4} \quad U_{4}=o(1) \text {. }
$$

Les formules (8) et $(10)_{1 ; 2,3,4}$ entrainent $I_{n} \rightarrow 0$, c. q. f. d.

3. D'une manière analogue on démontre les deux propositions suivantes:

Th é o r è m e l'. Si l'on désigne par $\bar{S}_{n}(x)$ les sommes partielles de la série

$$
\sum_{k=1}^{\infty}\left(-b_{k} \cos k x+a_{k} \sin k x\right)
$$

conjuguée à la série (1), on a presque partout

$$
\lim _{n \rightarrow \infty} \frac{1}{n} \sum_{n=1}^{n} \bar{S}_{n^{2}}(x)=-\frac{1}{\pi} \int_{0}^{\pi} \frac{f(x+t)-f(x-t)}{2 \operatorname{tg} \frac{1}{2} t} d t .
$$

Th é or è m e I". Si la série (1) est une série de Fourier-Stieltjes, c. à. d. si

$$
a_{n}+i b_{n}=\frac{1}{\pi} \int_{0}^{2 \pi} e^{i n t} d F(t)
$$

$F(t)$ étant une fonction réelle d̀ variation bornée, on a presque partout

$$
\lim _{n \rightarrow \infty} \frac{1}{n} \sum_{v=1}^{n} S_{r^{2}}(x)=F^{\prime}(x) .
$$

Dans la démonstration du théorème $I^{\prime \prime}$ il faut remplacer l'intégrale $I_{n}$ par

$$
\begin{gathered}
I_{n}^{*}=\frac{1}{n \pi} \int_{0}^{\pi} \frac{H_{n}(t)}{2 \sin \frac{1}{2} t} d \psi(t), \\
\text { où } \left.\psi(t)=\psi_{x}(t)=F(x+t)-F(x-t)-2 t F^{\prime}(x)^{4}\right) .
\end{gathered}
$$

3) Cela revient à constater que les coefficients de Fourier-Lebesgue tendent vers zéro. p. $59-60$.

). Voir p. ex.: A, Zygmund, Trigonometrical series (Warszawa 1935) 
Le raisonnement qui a nous servi pour démontrer que $U_{4} \longrightarrow 0$ ne s'applique pas ici, car les coefficients de Fourier-Stieltjes ne tendent pas en général vers zéro. Mais nous pouvons faire appel à un résultat dû à MM. HARDY et LitTLEwood ${ }^{5}$ ):

Si $t(0<t<\pi)$ est un nombre incommensurable avec $\pi$, alors

$$
\sum_{n=1}^{n} e^{i r^{2} t}=o(n)
$$

Ceci posé, la démonstration du fait $U_{4}^{*} \longrightarrow 0$ n'offre pas des difficultés.

4. Thé or è m e II. Si $f(x)$ est une fonction à carré sommable, on a presque partout.

$$
\lim _{n \rightarrow \infty} \frac{1}{n} \sum_{v=1}^{n}\left|S_{p_{y}}(x)-f(x)\right|=0,
$$

$\left\{p_{v}\right\}$ étant une suite croissante des nombres entiers positifs, vérifiant la condition

$$
p_{k}^{2} \sum_{v=k}^{\infty} \frac{1}{v p_{v}^{2}}=O(1) \text { pour } k \rightarrow \infty .
$$

Remarquons que toute suite $\left\{p_{v}\right\}$ croissante et convexe remplit la condition $(\mathrm{T})$.

Démonstration. Quand $p_{r}=\nu$, la proposition se réduit à un théorème connu de MM. Hardy-LitTLewOod ${ }^{6}$ ). M. ZYYGund ${ }^{7}$ ) en a donné une démonstration simple et élégante et son raisonnement s'applique aussi au cas actuel. Pour la commodité du lecteur nous exposerons la démonstration in extenso. Posons

Comme

$$
\sigma_{n}(x)=\frac{S_{0}+S_{1}+\ldots+S_{n}}{n+1} .
$$

$\frac{1}{n} \sum_{v=1}^{n}\left|S_{p_{v}}(x)-f(x)\right| \leqslant \frac{1}{n} \sum_{r=1}^{n}\left|S_{p_{v}}(x)-\sigma_{p_{v}}(x)\right|+\frac{1}{n} \sum_{v=1}^{n}\left|\sigma_{p_{v}}(x)-f(x)\right|$,

5) G. H. Hardy and J. E. Littlewood, Some problems of Diophantine Approximation. II., Acta Math. 37 (1914) p. 193-239; p. 213, Theorem 2.14. 9) G. H. Hardy et J. E. Littlewood, Sur la série de Fourier d'une fonction à carré sommable, C. R. Acad. Sc. Paris 156 (1913) p. 1307-1309.

7) L. c. 1), p. 241 .

$$
\frac{1}{n} \sum_{v=1}^{n}\left|S_{p_{r}}-\sigma_{p_{r}}\right| \leqslant\left\{\frac{1}{n} \sum_{v=1}^{n}\left(S_{p_{r}}-\sigma_{p_{v}}\right)^{2}\right\}^{1 / 2}
$$

et $\lim _{v \rightarrow \infty}\left|\sigma_{p_{y}}(x)-f(x)\right|=0$ presque partout, il suffit de démontrer que

$$
\lim _{n \rightarrow \infty} \frac{1}{n} \sum_{v=1}^{n}\left[S_{p_{1}}(x)-\sigma_{p_{v}}(x)\right]^{2}=0 \text { presque partout. }
$$

Cette rélation est à son tour une conséquence immédiate de la convergence de la série

$$
\left.\sum_{v=1}^{\infty} \frac{1}{v^{\prime}}\left[S_{p_{v}}(x)-\sigma_{p_{1}}(x)\right]^{2}{ }^{8}\right)
$$

Une série de fonctions positives étant intégrable terme à terme il suffit de prouver que

$$
B=\sum_{v=1}^{\infty} \frac{1}{v} \int_{u}^{2 \pi}\left(S_{p_{v}}-\sigma_{p_{v}}\right)^{2} d x<\infty .
$$

Or,

où

$$
\begin{aligned}
& B=\sum_{v=1}^{n} \frac{1}{v} \cdot \frac{\pi}{\left(p_{v}+1\right)^{2}} \sum_{m=1}^{p_{v}} d_{m}^{2} m^{2}, \text { où } d_{m}^{2}=\alpha_{m}^{2}+b_{m}^{2}, \\
& \left.\quad \sum_{m=1}^{p_{r}} d_{m}^{2} m^{2}=\sum_{k=1}^{v} \underset{m=p_{k-1}+1}{\sum_{k}} d_{m}^{2} m^{2}\right) \\
& \quad \leqslant \sum_{k=1}^{v} p_{k}^{2} \cdot \sum_{m=p_{k-1}+1}^{p_{k}} d_{m}^{2}=\sum_{k=1}^{r} p_{k}^{2} \omega_{k} \quad\left(p_{0}=0\right),
\end{aligned}
$$

$$
w_{k}=\sum_{m=p_{k-1}+1}^{p_{k}} d_{m}^{2}
$$

(12), (13), (14) et la condition (T) donnent

$$
\begin{gathered}
B \leqslant \sum_{v=1}^{\infty} \frac{\pi}{v} \cdot \frac{1}{p_{v}^{2}} \sum_{k=1}^{v} p_{k}^{2} \omega_{k}=\pi \sum_{k=1}^{\infty} p_{k}^{2} \omega_{k} \cdot \sum_{v=k}^{\infty} \frac{1}{\nu p_{v}^{2}} \\
\leqslant \sum_{k=1}^{\infty}{ }_{\left({ }^{\prime}\right.}, M=M \sum_{k=1}^{\infty} d_{k}^{2}<\infty \quad(M-\text { une constante }) .
\end{gathered}
$$

Ainsi la prémière partie de notre énoncé est démontrée.

8) Si la série $\sum_{v=1}^{\infty} a_{v}$ converge, $a_{1}+2 a_{2}+\ldots+n a_{n}=o(n)$. 
Il reste de prouver que toute suite $\left\{p_{v}\right\}$ croissante et convexe remplit la condition (T). On a $p_{2 k}-p_{k} \geqslant p_{k}-p_{1}, \quad p_{2 k} \geqslant 2 p_{k}-p_{1} \geqslant C p_{k}$ pour $k \geqslant k_{0}, C=1,5$, donc

$$
p_{2^{m} k} \geqslant C^{m} p_{k} \text { pour } k \geqslant k_{0} \quad(m=1,2,3, \ldots) ;
$$

il s'ensuit pour $k \geqslant k_{0}$

$$
\begin{gathered}
p_{k}^{2} \sum_{v=k}^{\infty} \frac{1}{v p_{v}^{2}}=\frac{1}{k}+p_{k}^{2}\left(\sum_{v=k+1}^{2 k}+\sum_{v=2 k+1}^{4 k}+\ldots\right) \\
\leqslant 1+\log 2\left(1+C^{-2}+C^{-4}+\ldots\right)=M<\infty .
\end{gathered}
$$

5. En terminant j'indique quelques questions qui se rattachent aux théorèmes démontrés:

$1^{\circ}$ dans le théorème $\mathrm{I}$, peut-on remplacer la suite $\left\{S_{r^{n}}\right\}$ $\operatorname{par}\left\{S_{v: 3}\right\}$ ou $\left\{S_{v+4}\right\}$ etc.?

$2^{\circ}$ le théorème II est-il vrai pour toute suite croissante $\left\{p_{v}\right\}$ ? $a<1$ ?

$3^{\circ}$ peut - on remplacer la sommabilité $(C, 1)$ par $(C, \alpha)$, où

(Reçu par la Rédaction le 11. 2. 1936). 